\title{
Controle de Euphorbia heterophylla com mesotrione e óleos para diferentes pontas de pulverização ${ }^{1}$
}

\section{Control of Euphorbia heterophylla with mesotrione and oils to different nozzles}

\author{
José Fernando Jurca Grigolli ${ }^{2}$, Fernanda Campos Mastrotti Pereira ${ }^{2}$, Luis Colina Peñaherrera ${ }^{2}$,
} Edson Aparecido dos Santos ${ }^{2}$, Marcelo da Costa Ferreira ${ }^{3}$

Resumo - O objetivo do trabalho foi avaliar a interação entre adjuvantes e pontas de pulverização no controle de Euphorbia heterophylla pelo herbicida mesotrione. Realizou-se a aplicação do herbicida mesotrione através de três modelos de pontas de pulverização (TTI 110. 02, AI 110.02 e TTI 110.02) e duas dosagens dos adjuvantes: óleo mineral e vegetal (0,5 e 3,0\%). Também foram verificados os atributos qualitativos das pontas, utilizando-se uma mesa de distribuição e um laser analisador de gotas. Determinou-se o controle visual nas plantas de leiteiro aos 2, 7, 14 e 21 dias após a aplicação, a massa seca da parte aérea das plantas aos 21 dias, a distribuição das gotas conferida pelas pontas, o DMV, a porcentagem do volume aplicado com gotas menores que $200 \mu \mathrm{m}$, o coeficiente de variação (CV) do espaçamento entre as pontas e o coeficiente de uniformidade (SPAN). Doses maiores de óleo apresentaram melhor controle das plantas de leiteiro, independente da ponta utilizada. $\mathrm{O}$ óleo vegetal apresentou melhor desempenho em relação ao mineral. Os adjuvantes reduziram o DMV de todas as pontas, aumentaram a porcentagem de gotas menores que $200 \mu \mathrm{m}$ e reduziram os valores de SPAN para a ponta TT 110.02. A adição de adjuvantes possibilita maior uniformidade mesmo com o aumento do espaçamento das pontas na barra de pulverização para os modelos TT 110.02 e TTI 110.02 .

Palavras-chave: TT 110.02, AI 110.02, TTI 110.02, óleo vegetal, óleo mineral.

\begin{abstract}
The aim of this study was evaluate the interaction between adjuvants and spray nozzles Euphorbia heterophylla control with mesotrione. Was held application of the herbicide by three models of spray nozzles (TT 110.02, AI 110.02 and TTI 110.02) and two dosages of mineral and vegetal oil $(0.5$ and $3.0 \%)$. Also was verified the qualitative attributes of nozzles, using a distribution table and a droplet analyzer laser. We evaluated the visual intoxication in plants at 2, 7, 14 and 21 days after application, the dry mass of the plants at 21 days, the distribution of the drops given by the nozzles, the VDM, the percentage of volume applied with drops smaller than 200 microns, the coefficient of space variation (CV) between nozzles and the coefficient of uniformity (SPAN). Higher doses of oil were more damaging to dairy plants, with all spray nozzles tested. The vegetable oil performed better in relation to the mineral. The adjuvants reduced the DMV of all nozzles, increasing the percentage of droplets smaller than 200 microns and reduced SPAN values for TT 110.02 nozzle. The adjuvants addition enables greater

\footnotetext{
${ }^{1}$ Recebido para publicação em 13/09/2011 e aceito em 05/11/2011

${ }^{2}$ Pós-Graduando, FCAV/UNESP, Via de Acesso Prof. Paulo Donato Castellane, s/n, 14884-900, Departamento de Fitossanidade. E-mail: jose_fernando_jg@ yahoo.com.br

${ }^{3}$ Prof. Dr., FCAV/UNESP, Via de Acesso Prof. Paulo Donato Castellane, s/n, 14884-900, Departamento de Fitossanidade.
} 
Grigolli et al.

uniformity even with the increased spacing of nozzles (TT 110.02 and TTI 110.02) on the spray boom.

Keywords: TT 110.02, AI 110.02, TTI 110.02, vegetable oil, mineral oil.

\section{Introdução}

Uma das plantas daninhas que mais se destaca em agressividade nas lavouras brasileiras é o leiteiro (Euphorbia heterophylla). Esta, por sua vez contribui para reduções na produtividade de diversas culturas tradicionais, principalmente a cultura da soja (Kern et al., 2009). Sua importância como planta daninha aumentou com o surgimento de populações resistentes aos herbicidas inibidores da acetolactato sintase (ALS), largamente utilizados na soja (Vidal \& Fleck, 1997; Melhorança \& Pereira, 1999). Dessa forma, a busca por herbicidas com novos mecanismos de ação, bem como melhorias nas condições de aplicação, são imprescindíveis para o sucesso no controle desta planta daninha.

Nesse sentido, o uso da tecnologia de aplicação visa aumentar a eficiência e melhorar as condições operacionais. Fatores como pontas a serem utilizadas aliadas a adição de adjuvantes à calda são pontos amplamente discutidos. Essa associação pode influenciar diretamente a cobertura proporcionada ao alvo. A avaliação das características qualitativas como o diâmetro mediano volumétrico, amplitude de dispersão das gotas, potencial de deriva e coeficiente de variação da sobreposição dos jatos produzidos, servem como parâmetros para analisar a qualidade da aplicação (Etheridge et al., 1999; Miller et al., 2000; Ferreira et al., 2007).

Os resultados das pulverizações são variáveis nas lavouras, sendo que o grau de sucesso geralmente é determinado pela quantidade e uniformidade da cobertura (Cunha et al., 2006). Segundo os mesmos autores, a eficácia do tratamento depende não somente da quantidade de material depositado sobre a vegetação, mas também da uniformidade de cobertura do alvo. Para que haja sucesso na aplicação de produtos fitossanitários, é preciso atingir o alvo, e para se obter adequada cobertura da área pelo produto é necessário conhecer as características de deposição proporcionadas pelo equipamento, produto e forma de aplicação (Ferreira et al., 2009).

As pontas de pulverização são os componentes mais importantes de um pulverizador, pois são responsáveis pela determinação da vazão da calda, pela distribuição uniforme do produto fitossanitário sobre o alvo biológico, e pela formação das gotas de tamanho compatível com a finalidade a que se destinam (Mathews, 1979). Por isso, o conhecimento da distribuição quantitativa da calda pulverizada é de grande importância (Perecin et al., 1999).

Dessa forma, o controle destas características é ferramenta importante no manejo de plantas daninhas, sendo relatado por Costa et al. (2008) por meio da avaliação de pontas de pulverização, vazões e diâmetro de gotas. Os autores verificaram que para a dessecação do capim-braquiária as pontas XR 110.02 VS apresentaram melhor deposição, trabalhando em volume de $200 \mathrm{~L} \mathrm{ha}^{-1}$, em relação às pontas TX4 VS, TX8 VK, AI 110. 02VS e TJ 60 110.02VS. Os mesmos autores ainda destacam que pontas que produzem gotas muito finas podem provocar falhas no controle em função das perdas por deriva e evaporação.

A utilização de adjuvantes pode alterar o padrão de gotas e a vazão das pontas de pulverização. Essas alterações podem acarretar em modificações nas propriedades do líquido pulverizado, podendo influenciar tanto no processo de formação das gotas como o comportamento destas em contato com o alvo, 
Grigolli et al.

alterando o risco potencial de deriva da aplicação (Miller \& Butler Ellis, 2000).

O acréscimo de óleo é uma prática recomendada para muitos produtos fitossanitários, uma vez que confere redução da tensão superficial da calda, possibilitando melhorias na absorção do herbicida, redução de possíveis perdas e, consequentemente, otimização das práticas de manejo de plantas daninhas (Mendonça et al., 2007). Dessa forma, objetivou-se com o trabalho avaliar a interação entre adjuvantes e pontas de pulverização no controle de Euphorbia heterophylla e em parâmetros que influenciam diretamente na qualidade de aplicação.

\section{Material e métodos}

Os trabalhos foram realizados nos meses de Setembro e Outubro de 2010 no Laboratório de Análise de Partículas da UNESP, em Jaboticabal, SP. O delineamento experimental utilizado foi blocos casualizados, em esquema fatorial com 15 tratamentos (3 pontas de pulverização x 5 doses de adjuvantes) e quatro repetições.

As pontas utilizadas foram TT 110.02, AI 110.02 e TTI 110.02 e os adjuvantes óleo mineral - Assist $^{\circledR}(0,5$ e $3,0 \% \mathrm{v} / \mathrm{v})$, vegetal Veget'Oil $^{\circledR}(0,5$ e $3,0 \%$ v/v) e sem adjuvantes. $\mathrm{O}$ herbicida utilizado foi mesotrione (Callisto ${ }^{\circledR}$ - SC) na dosagem de 168 g i.a. ha ${ }^{-1}$. Os dados obtidos foram submetidos à análise de variância e as médias dos tratamentos comparadas por meio do teste de Tukey a $5 \%$ de probabilidade.

Controle de leiteiro por mesotrione em função de pontas de pulverização e adição de óleos

Sementes de leiteiro foram semeadas em vasos contendo $1,0 \mathrm{dm}^{3}$ de substrato composto por solo proveniente de horizonte B. Após a germinação e emergência das plantas, foram mantidas duas plantas por vaso até que estivessem no estádio de duas folhas verdadeiras. Neste momento foi aplicado $o$ mesotrione na dosagem de $168 \mathrm{~g}$ i.a. ha ${ }^{-1}$. Utilizou-se um pulverizador costal pressurizado $\left(\mathrm{CO}_{2}\right)$ com barra munida de duas pontas espaçadas em $0,5 \mathrm{~m}$, à altura de $0,5 \mathrm{~m}$ em relação ao alvo, com pressão de $40 \mathrm{lbf} \mathrm{pol}^{-}$ ${ }^{2}$, que forneceu um volume de calda equivalente a $100 \mathrm{~L} \mathrm{ha}^{-1}$. No início da aplicação as condições ambientais eram temperatura $27{ }^{\circ} \mathrm{C}$ e umidade relativa do ar $50 \%$. No final da aplicação, as condições ambientais eram temperatura $31^{\circ} \mathrm{C}$ e umidade relativa do ar $42 \%$. A aplicação foi realizada às 9:00 h e com velocidade do vento de $0,1 \mathrm{~m} \mathrm{~s}^{-1}$.

Os tratamentos consistiram na combinação das três pontas de pulverização e dois tipos de óleos (mineral Assist ${ }^{\circledR}$ e vegetal Veget'Oil $\left.^{\circledR}\right)$ em três doses cada $(0,0 ; 0,5$ e $3,0 \% \mathrm{em} \mathrm{v} / \mathrm{v})$. É importante mencionar que o produto comercial utilizado é recomendado com $0,5 \%$ de óleo mineral.

Foram realizadas avaliações de controle visual (EWRC, 1964) aos 2, 7, 14 e 21 dias após a aplicação (DAA), quando foi coletada a parte aérea das plantas (corte rente ao solo) e determinada a massa seca da parte aérea (MSPA) após secagem em estufa de circulação forçada de ar a $65^{\circ} \mathrm{C}$ por 96 horas. Para a análise estatística dos dados não foi aplicada nenhuma transformação.

\section{Distribuição, coeficiente de variação do jato aspergido e padrão de deposição de gotas em função de pontas e adjuvantes}

A distribuição conferida pela calda em função dos tratamentos foi avaliada em uma mesa, constituída de 67 canaletas em formato de "V", espaçadas entre si por $2,5 \mathrm{~cm}$. Cada ponta individualmente foi posicionada em um suporte a altura de $0,5 \mathrm{~m}$ em relação à canaleta central. As canaletas conduziam a calda para 
Grigolli et al.

coletores graduados (até 0,1 L) alinhados ao longo da faixa de deposição, onde foram coletados os valores de volumes correspondentes a cada canaleta após pulverização. Dessa forma, obteve-se a curva de deposição, possibilitando a determinação do coeficiente de variação (CV\%) de sobreposição dos jatos aspergidos com auxílio do programa Microsoft Excel ${ }^{\circledR}$.

Para avaliar o padrão de gotas produzidas, utilizou-se, em laboratório, um analisador de gotas em tempo real (Mastersizer $S^{\circledR}$, versão 2,15) da Malvern Instrumentes Ltd. Sua leitura é baseada na luz que sofre difração durante a passagem de gotas pulverizadas pela região de amostragem do aparelho (Schick, 1997). Foi avaliado o DMV, que possibilitou a determinação da percentagem de gotas mais propensas à deriva (valores de DMV inferiores a $200 \mu \mathrm{m})$. O DMV é caracterizado como o diâmetro da gota em que $50 \%$ do volume do líquido pulverizado é constituído por gotas de tamanho maior ou menor que esse valor. Além disso, avaliou-se também o coeficiente de uniformidade (SPAN). Para a análise estatística os valores obtidos de DMV, porcentagem de gotas com DMV inferior a $200 \mu \mathrm{m}$ e SPAN foram transformados em $\operatorname{arcsen} \sqrt{ }(\mathrm{X} / 100)$.

\section{Resultados e discussão}

Controle de leiteiro por mesotrione em função de pontas de pulverização e adição de óleos

Por meio da avaliação de controle visual provocada pelos tratamentos nas plantas de leiteiro, concluiu-se que aos 2 DAA houve efeito apenas dos óleos nas plantas (Tabela 1), visto que o uso de herbicidas associados a adjuvantes foi significativamente diferente do uso do herbicida sem adjuvantes. No entanto, independentemente da ponta, do tipo e da dose de óleo utilizado, não foram observadas variações nos danos foliares. O coeficiente de variação elevado nesta avaliação é justificado pelo grande número de plantas sem sintomas.

Já aos 7 DAA, para pontas TT 110.02, observou-se que o efeito da parceria mesotrione + óleo vegetal promoveu maior controle das plantas daninhas, sendo o uso de $3 \%$ de óleo vegetal o mais prejudicial às plantas (Tabela 1). Ainda, para essa ponta, os danos foram incrementados com o aumento da dose do óleo. O mesmo efeito foi observado para a ponta AI 110.02. Para ambas, o tratamento com menor concentração de óleo mineral teve efeito semelhante à aplicação de herbicida sem adjuvantes.

Aos 14 DAA a concentração dos óleos utilizados afetou o controle de leiteiro para a ponta TTI 110.02, que teve desempenho inferior as demais utilizando $0,5 \%$ de óleo vegetal ou mineral. Entretanto, o uso de 3\% de óleo vegetal incrementou o controle das plantas daninhas por todas as pontas testadas, alcançando 80,7 e $82,4 \%$ para as pontas TTI 110.02 e TT 110.02 .

Aos 21 DAA, embora sem diferenças significativas entre os tratamentos testados, o controle proporcionado pelos tratamentos oscilou entre 31,9 e $82,5 \%$. Entretanto, a análise dos dados de massa seca da parte aérea (MSPA) permite inferir que as maiores doses de adjuvantes promoveram maiores danos, ocasionando a morte de algumas plantas (Figura 1). Assim, os valores observados de MSPA foram menores à medida que a concentração dos óleos aumentou (Figura 2), fato que pode ser notado para todas as pontas testadas. A adição de óleo à calda, além de favorecer a absorção do herbicida e reduzir perdas, pode também afetar propriedades da folha, principalmente folhas jovens e túrgidas como as adotadas no experimento (Matuo, 1989). 
Grigolli et al.

Tabela 1. Controle de plantas de leiteiro até 21 dias após a aplicação de mesotrione com diferentes pontas e adjuvantes. Jaboticabal-SP, 2010.

\begin{tabular}{|c|c|c|c|c|c|c|c|c|c|c|}
\hline \multicolumn{3}{|c|}{ Tratamento } & \multicolumn{8}{|c|}{ Dias Após a Aplicação } \\
\hline Ponta & Óleo & $\mathrm{C}^{1}$ & & & & 7 & & 14 & & \\
\hline \multirow{5}{*}{ TT 110.02} & \multirow{2}{*}{ Mineral } & 0,5 & 0,0 & $\mathrm{Ab}$ & 28,2 & $\mathrm{Da}$ & 61,3 & $\mathrm{BCa}$ & 39,7 & $\mathrm{Aa}$ \\
\hline & & 3,0 & 2,5 & $\mathrm{Aa}$ & 42,8 & $\mathrm{BCb}$ & 65,5 & $\mathrm{ABCa}$ & 62,2 & $\mathrm{Aa}$ \\
\hline & \multirow{2}{*}{ Vegetal } & 0,5 & 5,0 & $\mathrm{Aa}$ & 55,3 & $\mathrm{Ba}$ & 74,7 & $\mathrm{ABa}$ & 64,6 & $\mathrm{Aa}$ \\
\hline & & 3,0 & 2,5 & $\mathrm{Aa}$ & 72,9 & $\mathrm{Aa}$ & 82,5 & $\mathrm{Aa}$ & 82,5 & Aa \\
\hline & \multicolumn{2}{|c|}{ Sem Adjuvante } & 0,0 & $\mathrm{Ab}$ & 29,7 & $\mathrm{CDa}$ & 49,4 & $\mathrm{Ca}$ & 43,7 & $\mathrm{Aa}$ \\
\hline \multirow{5}{*}{ AI 110.02} & \multirow{2}{*}{ Mineral } & 0,5 & 14,3 & $\mathrm{Aa}$ & 32,9 & $\mathrm{Ca}$ & 56,2 & $\mathrm{Bab}$ & 48,7 & $\mathrm{Aa}$ \\
\hline & & 3,0 & 5,0 & $\mathrm{Ba}$ & 43,9 & $\mathrm{BCb}$ & 65,7 & $\mathrm{ABa}$ & 55,3 & $\mathrm{Aa}$ \\
\hline & \multirow{2}{*}{ Vegetal } & 0,5 & 5,0 & $\mathrm{Aa}$ & 50,8 & $\mathrm{ABa}$ & 64,3 & $\mathrm{ABa}$ & 53,2 & $\mathrm{Aa}$ \\
\hline & & 3,0 & 2,5 & $\mathrm{Aa}$ & 62,0 & $\mathrm{Aa}$ & 77,7 & $\mathrm{Aa}$ & 66,4 & $\mathrm{Aa}$ \\
\hline & \multicolumn{2}{|c|}{ Sem Adjuvante } & 0,0 & $\mathrm{Bb}$ & 29,9 & $\mathrm{Ca}$ & 35,2 & $\mathrm{Ca}$ & 31,9 & $\mathrm{Aa}$ \\
\hline \multirow{5}{*}{ TTI 110.02} & \multirow{2}{*}{ Mineral } & 0,5 & 10,7 & $\mathrm{Aa}$ & 29,9 & $\mathrm{Ba}$ & 42,1 & $\mathrm{Bb}$ & 43,3 & $\mathrm{Aa}$ \\
\hline & & 3,0 & 3,2 & $\mathrm{Aa}$ & 58,9 & Aa & 72,1 & $\mathrm{Aa}$ & 62,8 & $\mathrm{Aa}$ \\
\hline & \multirow{2}{*}{ Vegetal } & 0,5 & 5,0 & $\mathrm{Aa}$ & 35,5 & $\mathrm{Bb}$ & 42,9 & $\mathrm{Bb}$ & 47,9 & $\mathrm{Aa}$ \\
\hline & & 3,0 & 5,0 & $\mathrm{Aa}$ & 71,4 & $\mathrm{Aa}$ & 80,7 & $\mathrm{Aa}$ & 72,4 & $\mathrm{Aa}$ \\
\hline & \multicolumn{2}{|c|}{ Sem Adjuvante } & 10,0 & $\mathrm{Aa}$ & 29,9 & $\mathrm{Ba}$ & 47,9 & $\mathrm{Ba}$ & 39,2 & $\mathrm{Aa}$ \\
\hline \multicolumn{3}{|c|}{ Sem Herbicida } & \multicolumn{2}{|c|}{0,00} & \multicolumn{2}{|c|}{0,00} & \multicolumn{2}{|c|}{0,00} & \multicolumn{2}{|c|}{0,00} \\
\hline \multicolumn{3}{|c|}{ Coeficiente de Variação (\%) } & \multicolumn{2}{|c|}{99,12} & \multicolumn{2}{|c|}{15,95} & \multicolumn{2}{|r|}{14,21} & \multicolumn{2}{|c|}{19,72} \\
\hline \multicolumn{3}{|l|}{ Teste F } & \multicolumn{2}{|c|}{$2,99 *$} & \multicolumn{2}{|c|}{$4,36^{*}$} & \multicolumn{2}{|c|}{$4,27 *$} & \multicolumn{2}{|c|}{$1,21^{\mathrm{ns}}$} \\
\hline
\end{tabular}

${ }^{1} \mathrm{C}-$ Concentração do adjuvante em \% (v/v).

ns - Interação não significativa; * - Interação significativa.

Letras maiúsculas comparam médias da mesma ponta em diferentes concentrações de adjuvante. Letras minúsculas comparam médias dentro da mesma concentração de adjuvante em diferentes pontas. Médias seguidas pela mesma letra não diferem entre si pelo teste de Tukey a $5 \%$ de probabilidade.

Ressalta-se que para estas comparações, (ponta e adjuvante) optou-se por não adicionar os valores referentes à testemunha sem imediatamente após a média a letra herbicida foram sempre diferentes dos demais. correspondente à comparação (Tabela 1).

Por não estarem agrupados nos parâmetros 
Grigolli et al.

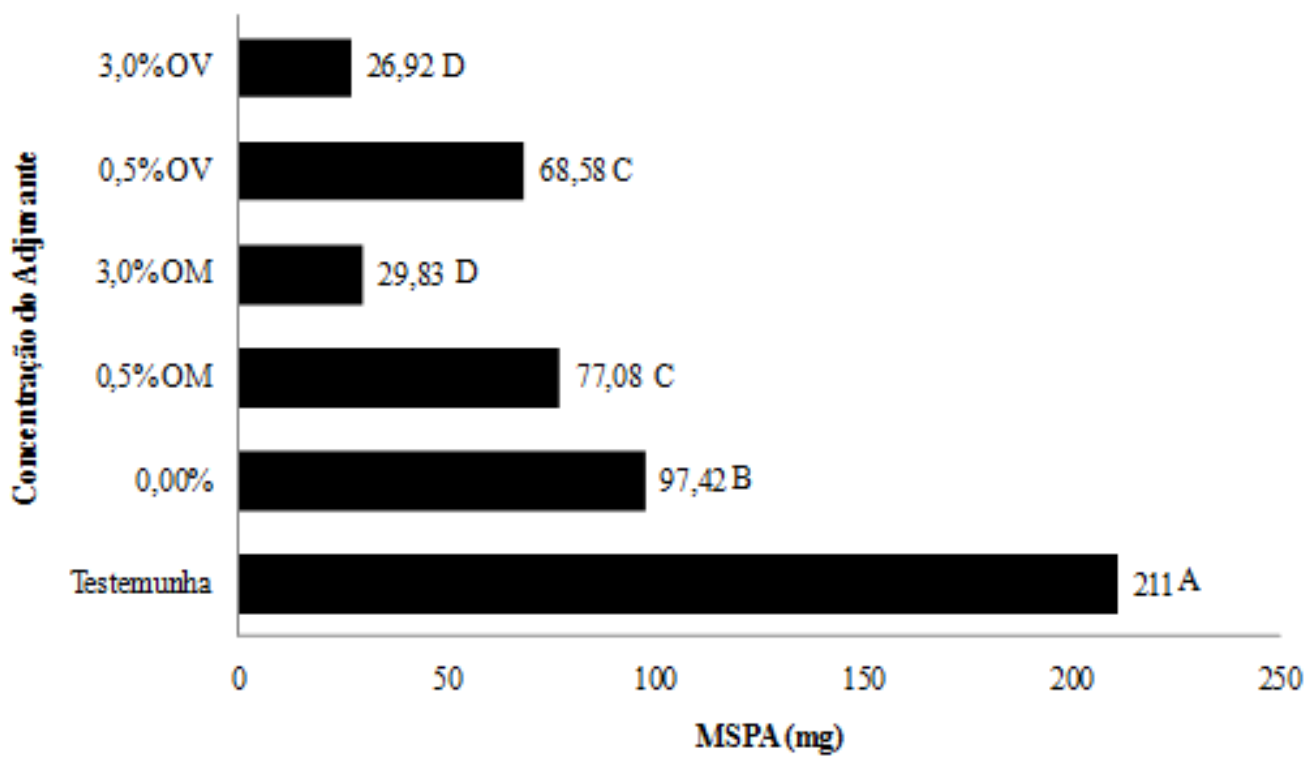

Médias seguidas pela mesma letra não diferem entre si pelo teste de Tukey a 5\% de probabilidade.

OM- óleo mineral; OV- óleo vegetal.

Figura 1. Valores de massa seca da parte aérea (MSPA) (mg) de plantas de leiteiro 21 dias após a aplicação de mesotrione com diferentes concentrações dos adjuvantes. Jaboticabal-SP, 2010.

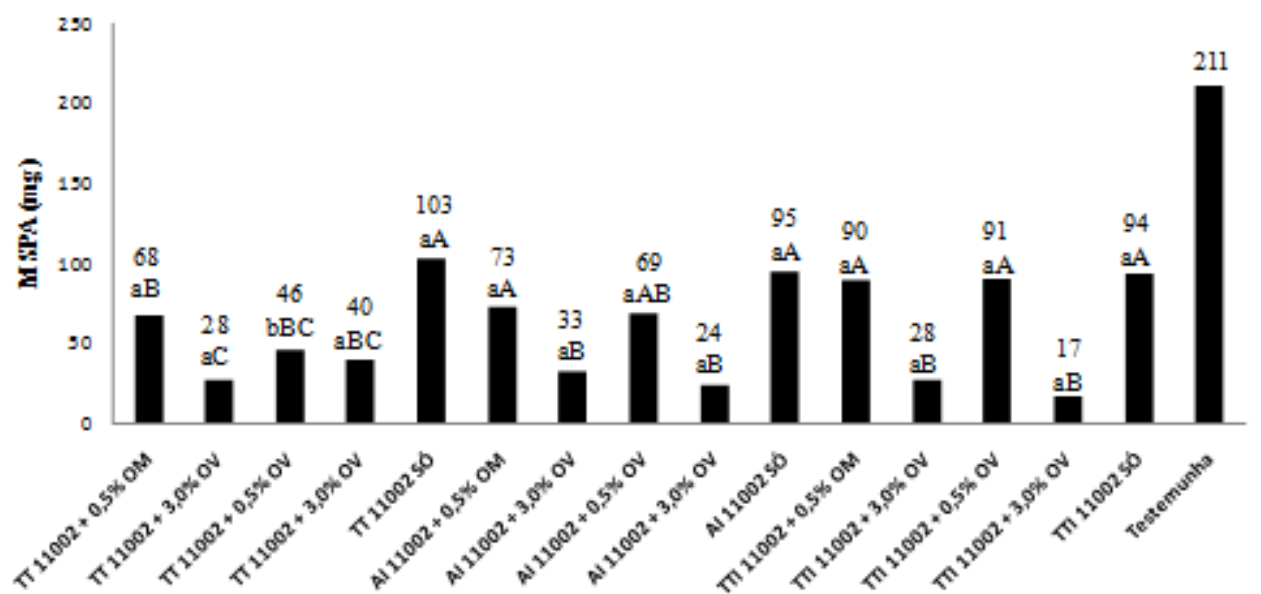

Letras maiúsculas comparam médias da mesma ponta em diferentes concentrações de adjuvante. Letras minúsculas comparam médias dentro da mesma concentração de adjuvante em diferentes pontas. Médias seguidas pela mesma letra não diferem entre si pelo teste de Tukey a $5 \%$ de probabilidade.

OM - óleo mineral; OV - óleo vegetal; SÓ - sem óleo.

Figura 2. Valores de massa seca da parte aérea (MSPA) (mg) de plantas de leiteiro 21 dias após a aplicação de mesotrione com diferentes pontas e adjuvantes. Jaboticabal-SP, 2010. 
Grigolli et al.

Distribuição, coeficiente de variação do jato aspergido e padrão de deposição de gotas em função das pontas e adjuvantes

Sobre o diâmetro mediano volumétrico (DMV), observou-se que a ausência dos óleos proporcionou valores maiores, assim como a adição de $0,5 \%$ de óleo mineral (Tabela 2). Foi observada redução de cerca de $20 \%$ do DMV com a adição de 3,0\% de óleo vegetal quando comparado ao tratamento sem adjuvantes, para a média de todas as pontas utilizadas. Entre as pontas com a mesma dose de óleo, observou-se que o modelo TT 110.02 apresentou menores valores de DMV em todas as concentrações dos adjuvantes em relação às outras.

Tabela 2. Variáveis qualitativas de pontas de pulverização, verificadas por meio de um analisador de gotas (Mastersizer $S^{\circledR}$, versão 2,15) em aplicação do herbicida mesotrione com adjuvantes. Jaboticabal-SP, 2010.

\begin{tabular}{|c|c|c|c|c|c|c|c|c|c|c|c|c|}
\hline \multicolumn{13}{|c|}{ DMV } \\
\hline \multirow{3}{*}{ Pontas } & \multicolumn{10}{|c|}{ Adjuvantes } & \multirow{2}{*}{\multicolumn{2}{|c|}{ Médias }} \\
\hline & \multirow{2}{*}{\multicolumn{2}{|c|}{ Sem }} & \multicolumn{4}{|c|}{ Óleo Mineral } & \multicolumn{4}{|c|}{ Óleo Vegetal } & & \\
\hline & & & \multicolumn{2}{|c|}{$0,5 \%$} & \multicolumn{2}{|c|}{$3,0 \%$} & \multicolumn{2}{|c|}{$0,5 \%$} & \multicolumn{2}{|c|}{$3,0 \%$} & & \\
\hline TT 110.02 & 217,92 & $\mathrm{Ac}$ & 195,56 & $\mathrm{ABc}$ & 174,13 & $\mathrm{BCc}$ & 169,10 & $\mathrm{Cc}$ & 158,86 & $\mathrm{Cb}$ & 183,12 & $\mathrm{c}$ \\
\hline AI 110.02 & 592,68 & $\mathrm{Ab}$ & 610,72 & $\mathrm{Ab}$ & 519,96 & $\mathrm{Cb}$ & 545,73 & $\mathrm{Bb}$ & 524,82 & $\mathrm{BCa}$ & 558,78 & b \\
\hline TTI 110.02 & 722,76 & $\mathrm{Aa}$ & 711,26 & $\mathrm{Aa}$ & 618,25 & $\mathrm{Ba}$ & 635,50 & $\mathrm{Ba}$ & 536,96 & $\mathrm{Ca}$ & 644,95 & $\mathrm{a}$ \\
\hline Média & 511,12 & $\mathrm{~A}$ & 505,85 & $\mathrm{~A}$ & 437,45 & $\mathrm{~B}$ & 450,11 & $\mathrm{~B}$ & 406,88 & $\mathrm{C}$ & & \\
\hline \multicolumn{13}{|c|}{$\mathrm{CV}(\%)=2,10$} \\
\hline \multicolumn{13}{|c|}{ SPAN } \\
\hline \multirow{2}{*}{ Pontas } & \multicolumn{10}{|c|}{ Adjuvantes } & \multirow{2}{*}{\multicolumn{2}{|c|}{ Médias }} \\
\hline & \multicolumn{2}{|c|}{ Sem } & \multicolumn{2}{|c|}{$0,5 \% \mathrm{OM}$} & \multicolumn{2}{|c|}{$3,0 \% \mathrm{OM}$} & \multicolumn{2}{|c|}{$0,5 \% \mathrm{OV}$} & \multicolumn{2}{|c|}{$3,0 \% \mathrm{OV}$} & & \\
\hline TT 110.02 & 2,59 & $\mathrm{Aa}$ & 1,67 & $\mathrm{Ba}$ & 1,39 & $\mathrm{Ba}$ & 1,47 & $\mathrm{Ba}$ & 1,39 & $\mathrm{Ba}$ & 1,70 & $\mathrm{a}$ \\
\hline AI 110.02 & 1,57 & $\mathrm{Ab}$ & 1,83 & $\mathrm{Aa}$ & 1,45 & $\mathrm{Aa}$ & 1,50 & $\mathrm{Aa}$ & 1,63 & $\mathrm{Aa}$ & 1,59 & $a b$ \\
\hline TTI 110.02 & 1,25 & $\mathrm{Ab}$ & 1,38 & $\mathrm{Aa}$ & 1,40 & $\mathrm{Aa}$ & 1,38 & $\mathrm{Aa}$ & 1,53 & $\mathrm{Aa}$ & 1,39 & $\mathrm{~b}$ \\
\hline Média & 1,80 & $\mathrm{~A}$ & 1,63 & $\mathrm{~A}$ & 1,41 & $\mathrm{~A}$ & 1,45 & $\mathrm{~A}$ & 1,52 & $\mathrm{~A}$ & & \\
\hline \multicolumn{13}{|c|}{$\mathrm{CV}(\%)=20,17$} \\
\hline \multicolumn{13}{|c|}{$\%$ Gotas $<200 \mu \mathrm{m}^{1}$} \\
\hline \multirow{2}{*}{ Pontas } & \multicolumn{10}{|c|}{ Adjuvantes } & \multirow{2}{*}{\multicolumn{2}{|c|}{ Médias }} \\
\hline & \multicolumn{2}{|c|}{ Sem } & \multicolumn{2}{|c|}{$0,5 \% \mathrm{OM}$} & \multicolumn{2}{|c|}{$3,0 \% \mathrm{OM}$} & \multicolumn{2}{|c|}{$0,5 \% \mathrm{OV}$} & $3,0 \%$ & $\mathrm{OV}$ & & \\
\hline TT 110.02 & 43,48 & $\mathrm{Da}$ & 47,42 & $\mathrm{Ca}$ & 52,63 & $\mathrm{Ba}$ & 53,59 & $\mathrm{ABa}$ & 56,11 & $\mathrm{Aa}$ & 50,65 & $\mathrm{a}$ \\
\hline AI 110.02 & 17,32 & $\mathrm{Bb}$ & 16,98 & $\mathrm{Bb}$ & 21,31 & $\mathrm{Ab}$ & 21,06 & $\mathrm{Ab}$ & 22,98 & $\mathrm{Ab}$ & 19,93 & $\mathrm{~b}$ \\
\hline TTI 110.02 & 11,60 & $\mathrm{Cc}$ & 13,38 & $\mathrm{BCc}$ & 15,92 & $\mathrm{Bc}$ & 15,26 & $\mathrm{Bc}$ & 19,37 & $\mathrm{Ac}$ & 15,11 & $\mathrm{c}$ \\
\hline Média & 24,13 & $\mathrm{C}$ & 25,93 & $\mathrm{C}$ & 29,95 & $\mathrm{~B}$ & 29,97 & $\mathrm{~B}$ & 32,82 & $\mathrm{~A}$ & & \\
\hline $\mathrm{CV}(\%)=4$ & & & & & & & & & & & & \\
\hline
\end{tabular}

A adição de ambos os óleos em mineral para a ponta AI 110.02 (Tabela 2). concentrações crescentes reduziram Esses resultados corroboram com os obtidos significativamente o DMV em todas as pontas por Matuo et al. (1989), onde os adjuvantes avaliadas, com exceção do uso de $0,5 \%$ de óleo Aterbane, Energic, OED-Green e Joint 
Grigolli et al.

proporcionaram um menor DMV em relação aos tratamentos sem adjuvantes. Além disso, as pontas utilizadas no ensaio apresentam maior predisposição para soluções contendo adjuvantes e possibilidade de redução do tamanho de gotas (Butler Ellis, 2004).

A ponta TT 110.02 apresentou valores de DMV inferiores a $200 \mu \mathrm{m}$ em todos os tratamentos. Geralmente valores inferiores a esse indicam risco potencial de deriva, principalmente devido à presença de gotas menores do que $100 \mu \mathrm{m}$ (Cunha et al. 2003). As pontas AI 110.02 e TTI 110.02 apresentaram valores de DMV superiores a 500 $\mu \mathrm{m}$ em todos os tratamentos. Valores acima de $500 \mu \mathrm{m}$ podem acarretar problemas de escorrimento, que ocorrem comumente com gotas maiores que $800 \mu \mathrm{m}$ (Cunha et al., 2003). As gotas produzidas a partir de pontas com indução de ar, entretanto, não são gotas maciças, mas com mesmo diâmetro, possuindo menor massa e menor velocidade, ficando menos suscetíveis ao ricocheteio e ao escorrimento. Devido ao seu tamanho extremamente grosso, a cobertura e distribuição das gotas pela superfície onde se depositaram é notadamente menor que a verificada para gotas finas, comprometendo a ação de produtos pouco sistêmicos. Assim, estas pontas de pulverização devem ser utilizadas criteriosamente, possibilitando redução dos riscos de deriva e escorrimento.

Observando-se o coeficiente de uniformidade (SPAN), verifica-se que a ponta TT 110.02 apresentou maiores valores sem adjuvantes em relação às outras pontas e também aos tratamentos com óleo mineral e vegetal (Tabela 2). Não houve diferença significativa quanto à adição de óleo mineral ou vegetal. Estes resultados corroboram com os obtidos por Camara et al. (2008) e Cunha et al. (2003), que verificaram que o uso de adjuvantes reduziu os valores do SPAN, indicando maior uniformidade no espectro do tamanho de gotas. No entanto, não foram observadas diferenças significativas entre as doses aplicadas.

Em relação à percentagem de gotas menores que $200 \mu \mathrm{m}$ para a ponta TT 110.02 , houve maior proporção em todas as concentrações de óleo, seguida pela ponta AI 110.02 e TTI 110.02, respectivamente. Deve-se ressaltar que a adição do óleo vegetal ou mineral aumentou o percentual dessas gotas em todas as pontas. Quanto menor essa percentagem, menor o risco de deriva do produto fitossanitário durante a aplicação. No entanto não existe um valor-padrão indicativo de deriva ou de aplicação segura. Assim, a adição de um óleo mineral ou vegetal a calda de pulverização deve ser feita criteriosamente, uma vez sua adição aumentou o risco de deriva, principalmente para modelos de pontas de pulverização que produzem gotas maciças. Foi verificada porcentagem superior ao dobro de gotas menores que $200 \mu \mathrm{m}$ para o modelo TT 110.02 em relação aos modelos com indução de ar. Como se verificou maior intoxicação das plantas daninhas - e consequentemente melhor controle - pelo herbicida mesotrione fazendo uso de pontas com indução de ar, pode-se afirmar que esses modelos possuem melhores atributos para serem indicados, ressalvadas as condições experimentais.

A curva de deposição de gotas foi utilizada para o cálculo do Coeficiente de Variação (CV). Na análise do CV verifica-se que as pontas sem adição de óleo apresentaram menor uniformidade entre os tratamentos avaliados. As pontas TT 110.02 (Figura 3A) e TTI 110.02 (Figura 3C) apresentaram valores de $\mathrm{CV}$ iguais a 7\%, atendendo as recomendações do fabricante para espaçamentos de até $0,6 \mathrm{~m}$. Contudo, o espaçamento pode ser ampliado para até $0,9 \mathrm{~m}$ com uso de adjuvante nas concentrações avaliadas. A ponta AI 110.02 (Figura 3B) apresentou desempenho próximo ao limite recomendado pelo fabricante $(0,4$ e $0,5 \mathrm{~m})$ 
Grigolli et al.

mesmo com uso de adjuvantes, mas não atendeu o critério de $7 \%$ do $\mathrm{CV}$ para a pressão avaliada. Resultados semelhantes foram obtidos por Camara et al. (2008) ao analisar a deposição individual de pontas utilizando apenas água e água em mistura a adjuvante sob diferentes pressões.

Visto o anteriormente exposto, pode ser notado que a adição de ambos os óleos testados reduziu significativamente o DMV para todas as pontas de pulverização avaliadas. O SPAN foi reduzido significativamente para a ponta TT 110.02 , e a percentagem de gotas menores que
$200 \mu \mathrm{m}$ tornou-se maior para todas as pontas na presença dos óleos.

Contudo, a utilização dos adjuvantes e pontas avaliadas deve ser feita com critérios, já que em alguns casos houve aumento do risco de deriva, que se agrava com a adição de 3,0\% de óleo vegetal, já que a dosagem incrementou significativamente a percentagem de gotas menores que $200 \mu \mathrm{m}$. Também foi notável que a adição de adjuvantes possibilitou o aumento do espaçamento das pontas na barra de pulverização para os modelos TT 110.02 e TTI 110.02 .
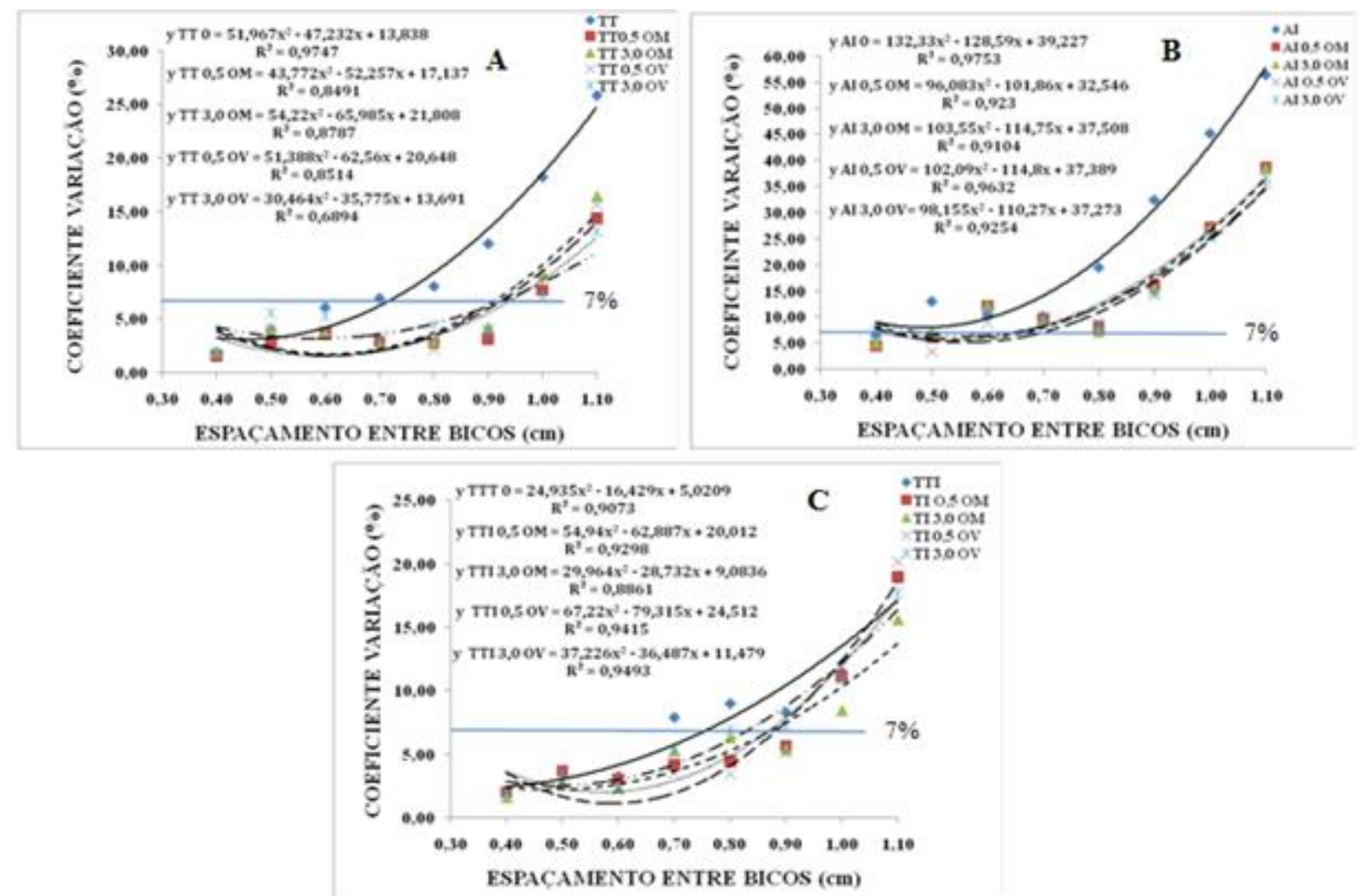

Figura 3. Coeficiente de Variação das pontas TT 110.02 (A), AI 110.02 (B) e TTI 110.02 (C) em aplicação de mesotrione com óleos. Jaboticabal-SP. 2010. 
Grigolli et al.

É importante ressaltar que o uso da ponta TT 110.02 com os adjuvantes testados é bastante recomendada para produtos de contato, uma vez que o tratamento apresentou grande proporção de gotas finas e médias, propiciando melhor cobertura a planta daninha alvo.

A adição de adjuvantes potencializou o efeito do herbicida utilizado em todas as pontas, sendo, portanto recomendado para o controle efetivo do leiteiro. Dosagens maiores de óleo foram mais danosas independentemente da ponta utilizada. Também foi perceptível que o comportamento do óleo vegetal foi melhor quando comparado aos efeitos proporcionados pelo óleo mineral no controle da planta daninha.

Por fim, o modelo TTI 110.02 com $3,0 \%$ de óleo vegetal apresentou os melhores atributos para aplicar o herbicida mesotrione no controle de plantas de leiteiro (Euphorbia heterophylla), no estádio de duas folhas verdadeiras.

\section{Conclusões}

A adição de óleos testados reduz o DMV para todas as pontas, o SPAN para a ponta TT 110.02, e aumenta a percentagem de gotas menores que $200 \mu \mathrm{m}$ para todas as pontas.

A adição de adjuvantes potencializa o efeito do herbicida utilizado em todas as pontas, sendo que maiores concentrações de óleo proporcionam maior controle nas plantas de leiteiro.

O óleo vegetal apresenta melhor controle de leiteiro do que o óleo mineral. A ponta TT 110.02 com 3,0\% de óleo vegetal apresenta os melhores resultados no controle de leiteiro com o herbicida mesotrione.

\section{Referências}

BUTLER ELLIS, M.C. The effect of spray liquid on the application of pesticide spray. In: RAETANO, C.G.; ANTUNIASSI, U.R. Qualidade em tecnologia de aplicação. Botucatu: FEPAF, 2004. p.167-176.

CAMARA, F.T. et al. Distribuição volumétrica e espectro de gotas de bicos hidráulicos de jato plano de faixa expandida XR 11003. Engenharia Agrícola, v.28, n.4, p.740-749, 2008 .

COSTA, N.V. et al. Efeito de pontas de pulverização na deposição e na dessecação em plantas de Brachiaria brizantha. Planta Daninha, v.26, n.4, p.923-933, 2008.

CUNHA, J.P.A.R. et al. Avaliação de estratégias para redução da deriva de agrotóxicos em pulverizações hidráulicas. Planta Daninha, v.21, n.2, p.325-332, 2003.

CUNHA, J.P.A.R.; REIS, E.F.; SANTOS, R.O. Controle químico da ferrugem asiática da soja em função de ponta de pulverização e de volume de calda. Ciência Rural, v.36, n.5, p.1360-1366, 2006.

ETHERIDGE, R.E. et al. Characterization of the spray droplet spectra and patterns of four venturi-type drift reduction nozzles. Weed Technology, v.13, n.4, p.765-70, 1999.

EWRC - EUROPEAN WEED RESEARCH COUNCIL. Report of $3^{\text {rd }}$ and $4^{\text {th }}$ meetings of EWRC. Cittee of methods in weed research. Weed Research, v.4, n.1, p.88, 1964.

FERREIRA, M.C.; DI OLIVEIRA, J.R.G.; DAL PIETRO, I.R.P.S. Distribuição da calda herbicida por pontas de pulverização agrícola utilizadas em áreas de reflorestamento com eucalipto. Engenharia Agrícola, v.29, n.2, p.267-276, 2009.

FERREIRA, M.C. et al., Fatores qualitativos da ponta de energia hidráulica ADGA 110015 


\section{Grigolli et al.}

para pulverização agrícola. Engenharia Agrícola, v. 27, n.2, p.471-478, 2007.

KERN, K.A. et al. The phytotoxic effect of exogenous ethanol on Euphorbia heterophylla L. Plant Physiology and Biochemistry, v.47, n.2, p.1095-1101, 2009.

MATHEWS, G.A. Pesticide application methods. London: Longman, 1979, 334p.

MATUO, T. et al. Efeito de alguns adjuvantes da pulverização nas propriedades físicas do líquido. Summa Phytopathologica, v.15, n.2, p.163-173, 1989.

MELHORANÇA, A.L.; PEREIRA, F.A.R. Eficiência do herbicida lactofen no controle de Euphorbia heterophylla, resistente aos herbicidas inibidores da enzima acetolactato sintase (ALS). Documentos Embrapa Agropecuária Oeste, v.3, n.3, p.11-14, 1999.

MENDONÇA, C.G.; RAETANO, C.G.; MENDONÇA, C.G. Tensão superficial estática de soluções aquosas com óleos minerais e vegetais utilizados na agricultura. Engenharia Agrícola, v.27, n2, p.16-23, 2007.

MILLER, P.C.H.; BUTLER ELLIS, M.C. Effects of formulation on spray nozzle performance for applications from groundbased boom sprayers. Crop Protection, v. 19, n.8-10, p.609-615, 2000.

PERECIN, D. et al. Avaliação do desempenho de bicos para aplicação de herbicidas. Planta Daninha, v.17, n.1, p.345-354, 1999.

SHICK, R.J. An engineer's practical guide drop size. Wheatom: Spraying Systems, 1997, $28 \mathrm{p}$.

VIDAL, R.A.; FLECK, N.G. Three weed species with confirmed resistance to herbicides in Brazil. In: MEETING OF THE WEED SCIENCE SOCIETY OF AMERICA, WSSA. Abstracts... 1997. p.100. 